\title{
On Full-View Coverage in Camera Sensor Networks
}

\author{
Yi Wang and Guohong Cao \\ Department of Computer Science \& Engineering \\ The Pennsylvania State University, University Park \\ Email: \{yuw124, gcao\}@cse.psu.edu
}

\begin{abstract}
Camera sensors are different from traditional scalar sensors as different cameras from different positions can form distinct views of the object. However, traditional disk sensing model does not consider this intrinsic property of camera sensors. To this end, we propose a novel model called full-view coverage. An object is considered to be full-view covered if for any direction from 0 to $2 \pi$ (object's facing direction), there is always a sensor such that the object is within the sensor's range and more importantly the sensor's viewing direction is sufficiently close to the object's facing direction. With this model, we propose an efficient method for full-view coverage detection in any given camera sensor networks. We also derive a sufficient condition on the sensor density needed for full-view coverage in a random uniform deployment. Finally, we show a necessary and sufficient condition on the sensor density for full-view coverage in a triangular lattice based deployment.
\end{abstract}

\section{INTRODUCTION}

Traditional sensor networks measure scalar phenomena in the physical world. Camera sensor networks can retrieve much richer information in the form of images or videos, and hence provide more detailed and interesting data of the environment. Such networks promise a wide range of applications in surveillance, traffic monitoring, habitat monitoring, health care and even online gaming [2]. Because of the huge potential in applications, camera sensor networks have drawn much attention in the past few years [18], [23].

One fundamental research issue is how well the target field is monitored, which is referred to as the coverage problem in wireless sensor networks. Existing work on this problem suggests a very simple model on characterizing the coverage: an object is considered to be covered if it is within the sensor's sensing range, which can be a disk [9] or sector [8]. With this generic model, extensive studies have been devoted to the problem of how to achieve $k$-coverage over a given target area [15], [21], [12], [3], where $k$ is a predefined parameter indicating the desired number of sensors (coverage degree) covering each object.

However, camera sensors are different from traditional scalar sensors. Camera sensors may generate very different views of the same object if they are from different viewpoints. For example, a camera sensor placed in front of a person can obtain the face image, but it can only view his back if it is behind him. In fact, studies in computer vision show that the object is more likely to be recognized by the recognition system if the image is captured at or near the frontal viewpoint [7], i.e., if the object is facing straight to the camera. As the

This work was supported in part by National Science Foundation under grant CNS-0916171.

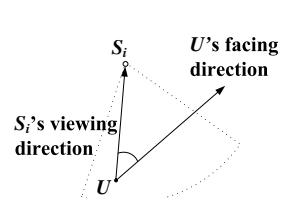

(a)

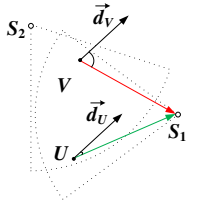

(b)
Fig. 1. (a) $U$ is an object; dotted line defines the sensing range of $S_{i}$ and $U S_{i}$ is its viewing direction; (b) Although $U$ and $V$ 's facing directions, $\overrightarrow{d_{U}}$ and $\overrightarrow{d_{V}}$, are the same, $S_{1}$ 's viewing direction is closer to $U$ 's facing direction.

angle between the object's facing direction and the camera's viewing direction (denoted by the vector from the object to the camera) increases (Fig. 1(a)), the detection rate drops dramatically [19], [17]. As a result, the viewing direction of the sensor has significant impact on the quality of coverage in a camera sensor network.

As none of the existing coverage models can be used to address the issues of viewing direction, we propose a novel model called full-view coverage. An object is considered to be full-view covered if no matter which direction the object faces, there is always a sensor whose sensing range includes the object and that sensor's viewing direction is sufficiently close to the object's facing direction (rigorous definition is given in Section II). Informally, if an area is full-view covered, it is guaranteed that every perspective of an object at any position is under the view of some camera sensor.

With this model, we study coverage issues arisen in camera sensor networks. One important problem is that given a deployed camera sensor network, how to determine if the target field is full-view covered? Compared with the traditional model, there are two factors that increase the complexity of the problem in full-view coverage. First, the sensing range of a camera sensor is a sector, which is supposed to be more complex than a disk. Second, and more importantly, the viewing direction of each camera sensor can vary from one position to another, and hence even if some objects are known to be covered (in traditional sense) by the same set of camera sensors, they may receive different quality of coverage due to position variance. For example, in Fig. 1(b), both objects $(U, V)$ are covered by camera sensors $S_{1}$ and $S_{2}$, and they are facing the same direction. However, the viewing direction of $S_{1}$ is closer to $U$ 's facing direction than to $V$ 's, meaning that $U$ receives better coverage (more likely to be recognized) than $V$. Moreover, there are infinite number of positions (points) to be considered in the target field and the object can face to any direction, which further increases the difficulty.

Another important problem is how to derive an estimate of the sensor density needed in a real deployment for full-view 


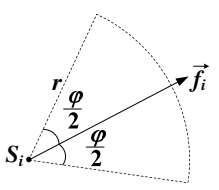

(a)

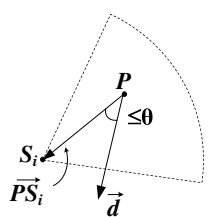

(b)
Fig. 2. The full-view coverage model

coverage. In practice, sensors can be either deployed randomly, e.g., being dropped from aircraft to an inaccessible zone, or deployed deterministically, e.g., being placed manually in a controlled environment. In both cases, a reliable estimation can serve as a guideline for the real deployment. Since most previous works mainly focus on disk sensing model, no result can be applied directly to full-view coverage, where combined effects of distance, camera's orientation and viewing direction make the geometric relationship between the objects and the sensors much more complex, and hence make the problem much more challenging.

Our contribution in this paper can be summarized as follows. First, we introduce a novel model that characterizes the intrinsic property of full-view coverage in a camera sensor network. Second, we propose an efficient method to deterministically detect if a target field can be full-view covered by any given set of camera sensors. Third, we derive an estimate of the sensor density needed for full-view coverage in a random deployment. Finally, we obtain a sufficient and necessary condition on the sensor density needed for full-view coverage in a triangle lattice based deployment and show that the density needed in this deployment pattern is no more than a factor of the density needed in any other deployment. To our best knowledge, we are the first to consider full-view coverage in camera sensor networks.

The remainder of this paper is structured as follows. Section II introduces the full-view coverage model. Section III gives the detailed description on full-view coverage detection for a given deployed camera sensor network. Section IV shows the density estimation for full-view coverage in a random deployment. Section V presents the density calculation result for full-view coverage in a triangular lattice deployment pattern. Section VI presents the numerical results regarding our estimation. The related work is reviewed in Section VII and the paper is concluded in Section VIII.

\section{NOTATIONS AND MODEL}

Camera sensors ${ }^{1}$ are deployed to monitor a bounded region $A$ (target field). Each sensor $S_{i}$ has a sensing range $r$, a fieldof-view (FoV) angle $\varphi$ and an orientation vector $\vec{f}_{i}$, which together define the sensing sector (Fig. 2(a)). We use $S_{i}$ to denote the $i$-th sensor. Without ambiguity, $S_{i}$ also denotes the sensor's position. For any two points $U, V$, let $\|U V\|$ denote the (Euclidean) distance between them. For any two vectors $\overrightarrow{v_{1}}$ and $\overrightarrow{v_{2}}$, let $\alpha\left(\overrightarrow{v_{1}}, \overrightarrow{v_{2}}\right)$ denote the angle between them, which ranges from 0 to $\pi$. A point $P$ is covered by a sensor $S_{i}$

\footnotetext{
${ }^{1}$ We may use cameras or sensors for short through out the paper.
}

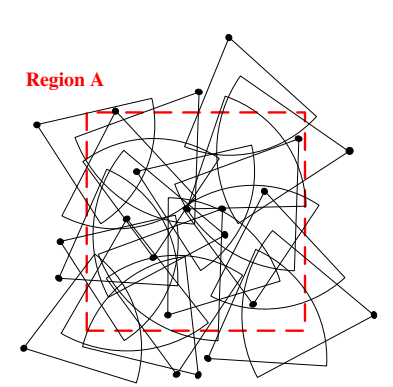

(a)

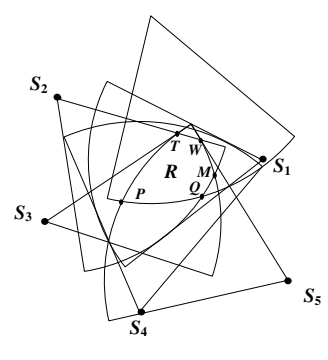

(b)
Fig. 3. (a) An example of a camera sensor network; how do we know if $A$ is full-view covered? (b) A sub-region $R$ whose boundary consists of 5 segments: $\overparen{T P}, \overparen{P Q}, \widehat{Q M}, \overparen{M W}$ and $\overline{W T}$.

if $P$ is in the sensing sector of $S_{i}$, i.e., $\left\|P S_{i}\right\|<r$ and $^{2}$ $\alpha\left(\overrightarrow{f_{i}}, \overrightarrow{S_{j} P}\right)<\varphi / 2$, where $\overrightarrow{S_{j} P}$ is the vector from $S_{j}$ to $P$.

Definition 2.1 (Full-View Coverage) A point $P$ is full-view covered if for any any vector $\vec{d}$ (the facing direction), there is a sensor $S_{i}$, such that $P$ is covered by $S_{i}$ and $\alpha\left(\vec{d}, \overrightarrow{P S_{i}}\right) \leq \theta$ (Fig. 2(b)). Here $\theta(\in[0, \pi / 2))$ is a predefined parameter which is called the effective angle. A region is full-view covered if every point in it is full-view covered.

\section{Full-View Coverage Detection}

In this section, we propose an efficient method to detect if the target region is full-view covered by a set of deployed camera sensors.

\section{A. Method overview}

Given a set of deployed sensors, region $A$ can be partitioned into sub-regions, where each sub-region is defined to be a set of points covered by the same set of sensors. The boundary of each sub-region consists of either segments of lines or arcs which are either part of the perimeter of the sensing sectors covering the sub-region or part of $A$ 's boundary. For example, in Fig. 3(b), sub-region $R$ is covered by 5 sensors and bounded by 5 segments: $\overparen{T P}, \overparen{P Q}, \overparen{Q M}, \widehat{M W}$ and $\overline{W T}$.

We first show that the whole region is full-view covered if and only if the boundary of each sub-region is full-view covered. Then the most tricky part is to determine if every point on a boundary segment is full-view covered, as there are still infinite number of positions to consider and the sensor's viewing direction vary from one position to another. To this end, we first show an equivalent condition on full-view coverage (Lemma 3.2), and then propose a novel method based on geometrical properties of the circumscribed circle and the inscribed angle (Lemma 3.3). The intuition is that if a point is full-view covered, there must be a set of sensors around it and the angle between the viewing directions of any two adjacent sensors is no more than $2 \theta$. For any two sensors, we actually identify the area (called safe region) in which for any point the angle between the two sensors' viewing directions is no more than $2 \theta$. Then we solve the detection problem by checking if the segment is contained in the safe region of every two adjacent sensors.

\footnotetext{
${ }^{2}$ For ease of analysis, we use " $<$ " instead of " $\leq$ ", although not essentially.
} 


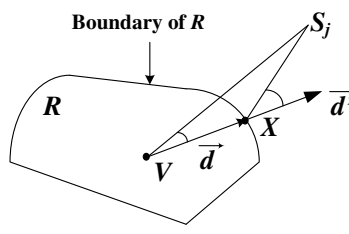

Fig. 4. Boundary condition.

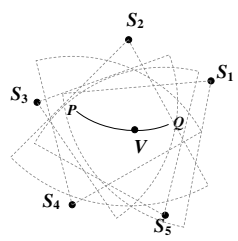

Fig. 5. The circular list of $V$

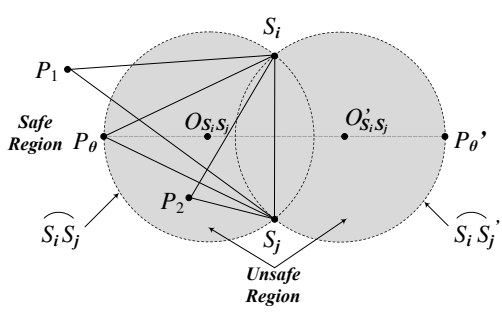

Fig. 6. The safe and unsafe region of $S_{i}$ and $S_{j}$.

\section{B. Detection Method Description}

We need to verify if the condition in Definition 2.1 holds for every point in $A$. Actually we only need to determine if it holds on the boundary of every sub-region in $A$.

Lemma 3.1 (Boundary Condition) The region $A$ is fullview covered if and only if the boundary of every sub-region is full-view covered by the given set of sensors.

Proof: The "only if" part is obvious. We only need to show the "if" part. We actually show that for a given subregion $R$, if $R$ 's boundary segments are all full-view covered, then $R$ is full-view covered.

Suppose there is an interior point $V \in R$ and a vector $\vec{d}$, such that for any sensor $S_{i}$ with $\left\|V S_{i}\right\| \leq r, \alpha\left(\vec{d}, \overrightarrow{V S}_{i}\right)>\theta$. Now consider the intersection point of $\vec{d}$ and $R$ 's boundary, which is denoted by $X$ (Fig. 4). We claim that $X$ is not full-view covered. In fact, consider a vector $\vec{d}^{\prime}$ which is parallel to $\vec{d}$ and originates from $X$. If $X$ is full-view covered, then there must be a sensor $S_{j}$ such that $\left\|X S_{j}\right\| \leq r$, $\alpha\left(\overrightarrow{d^{\prime}}, X \vec{S}_{j}\right) \leq \theta$. Clearly, $S_{j}$ also covers $V$. Furthermore, we have $\alpha\left(\vec{d}, V \overrightarrow{S_{i}}\right) \leq \alpha\left(\overrightarrow{d^{\prime}}, X \vec{S}_{j}\right) \leq \theta$, which is a contradiction. Therefore any interior point of $R$ is full-view covered if the boundary is full-view covered. The claim is proved.

Given a segment $\overline{P Q}$ on the boundary of a sub-region $R$, where $P$ and $Q$ are the two end points of the segment, we show a way to determine if every point on the segment is full-view covered. Note that every point on $\overline{P Q}$ is covered by the same set of sensors. For any point $V \in \overline{P Q}$, we can construct a circular list of these sensors regarding their viewing direction on $V$ as follows (Fig. 5). Initially the list is empty. We begin with any vector $\overrightarrow{V S_{i}}$ and place it into the list first. Then we rotate $\overrightarrow{V S_{i}}$ around $V$ in the counterclockwise direction until it becomes parallel to the next vector $\overrightarrow{V S_{j}}$. Then we place $\overrightarrow{V S_{j}}$ into the list, right after $\overrightarrow{V S}$. We continue rotating and placing vectors sequentially into the list until we see the beginning vector again. Then the list is completed. We denote the list by $C L_{V}=\left\{\overrightarrow{V S_{V_{1}}}, \ldots, \overrightarrow{V S_{V_{k}}}\right\}$, where $k$ is the number of sensors covering $\overline{P Q}$. Then the condition in Definition 2.1 is equivalent to the following.

Lemma 3.2 A given point $V$ is full-view covered if and only if for $C L_{V}$ constructed as above, the rotation angle from $\overrightarrow{V S_{V_{i}}}$ to $\overrightarrow{V S_{V_{i+1}}}$ is less than or equal to $2 \theta$ for any $1 \leq i \leq k$, where $V_{k+1}=V_{1}$.

Proof: Suppose the condition holds. Then for any $\vec{d}$, there are two sensor $S_{V_{i}}$ and $S_{V_{i+1}}$ such that either the rotation angle from $\overrightarrow{V S}$ to $\vec{d}$ or the angle from $\vec{d}$ to $\overrightarrow{V S_{V_{i+1}}}$ is less than or equal to $\theta$. Thus $V$ is full-view covered.
If $V$ is full-view covered but the rotation angle from $\overrightarrow{V S_{V_{i}}}$ to $\overrightarrow{V S_{V_{i+1}}}$ is larger than $2 \theta$ for some $i$. Then consider vector $\vec{d}$ along the bisector of the angle. The angle between either $\overrightarrow{V S_{V_{i}}}$ or $\overrightarrow{V S_{V_{i+1}}}$ and $d$ is larger than $\theta$. Therefore the condition is true.

We need to determine if the above condition holds for any $V \in \overline{P Q}$. To this end, we introduce the concept of safe region. For any two sensors $S_{i}$ and $S_{j}$, we define the safe region to be the area in which for any point $V, \alpha\left(\overrightarrow{V S_{i}}, \overrightarrow{V S_{j}}\right) \leq 2 \theta$; and define the unsafe region to be the area in which for any point $V, \alpha\left(\overrightarrow{V S_{i}}, \overrightarrow{V S_{j}}\right)>2 \theta$ (Fig. 6). The following lemma shows an efficient method to identify the two regions.

Lemma 3.3 Given $S_{i}$ and $S_{j}$, there are two $\operatorname{arcs} \widehat{S_{i} S_{j}}$ and ${\widehat{S_{i} S_{j}}}^{\prime}$ which connect $S_{i}$ and $S_{j}$ and are symmetrical with respect to line $S_{i} S_{j}$, such that the unsafe region is the enclosed region bounded by the arcs and the safe region is the open region outside the unsafe region.

Proof: We prove the lemma by showing how to find the two arcs. First we can find two different points $P_{\theta}$ and $P_{\theta}^{\prime}$ on the perpendicular bisector of segment $\overline{S_{i} S_{j}}$, such that $\angle S_{i} P_{\theta} S_{j}=\angle S_{i} P_{\theta}^{\prime} S_{j}=2 \theta$ and they are on different sides of $S_{i} S_{j}$. Without loss of generality, let $P_{\theta}$ be on the left side and $P_{\theta}^{\prime}$ be on the right side (Fig. 6).

We draw the circumscribed circles of triangle $\triangle S_{i} P_{\theta} S_{j}$ and $\triangle S_{i} P_{\theta}^{\prime} S_{j}$. Denote the centers of the circles by $O_{S_{i} S_{j}}$ and $O_{S_{i} S_{j}}^{\prime}$, and the radius (which is the same for both) by $r_{s a f e}$. Then arc $\overparen{S_{i} S_{j}}$ is the portion of the perimeter of $\odot O_{S_{i} S_{j}}$ on the left side and ${\widehat{S_{i} S_{j}}}^{\prime}$ is the portion of $\odot O_{S_{i} S_{j}}^{\prime}$ on the right.

In fact, for any circle and a fixed chord (defined here by $\overline{S_{i} S_{j}}$ ) of the circle, all inscribed angles with two endpoints at the ends of the chord are either equal or supplementary to each other. Specifically, they are equal if the third points of the angles are on the same side of the chord. Furthermore, for a given point $P_{\theta}$ on the perimeter of the circle and another point $P$ on the same side of line $S_{i} S_{j}$ as $P_{\theta}$, if $P$ is outside the circle $\left(\left\|P O_{S_{i} S_{j}}\right\|>r_{\text {safe }}\right)$, then $\angle S_{i} P S_{j}<\angle S_{i} P_{\theta} S_{j}$; if $P$ is inside the circle $\left(\left\|P O_{S_{i} S_{j}}\right\|<r_{\text {safe }}\right)$, then $\angle S_{i} P S_{j}>\angle S_{i} P_{\theta} S_{j}$. The proof of this property can be found in any textbook on Euclidean Geometry and hence omitted here.

Now we can give a necessary and sufficient condition for $\overline{P Q}$ to be full-view covered under some constraint.

Theorem 3.4 Suppose for every point $V \in \overline{P Q}$, the ordered list $C L_{V}=\left\{S_{V_{1}}, \ldots, S_{V_{k}}\right\}$ is the same (in a circular way). Then $\overline{P Q}$ is full-view covered if and only if it is within the polygon bounded by $\left\{\overline{S_{V_{i}} S_{V_{i+1}}}, 1 \leq i \leq k\right\}$ and for any $1 \leq$ 


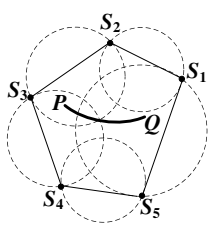

(a)

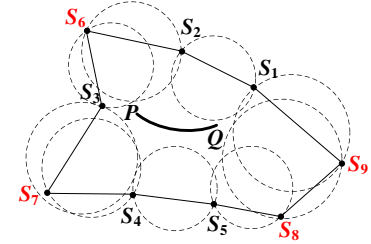

(b)
Fig. 7. (a) $\overline{P Q}$ is not full-view covered; (b) $\overline{P Q}$ is full-view covered.

$i \leq k$, the unsafe region of $S_{V_{i}}$ and $S_{V_{i+1}}$ does not intersect with $\overline{P Q}$, where $V_{k+1}$ denotes $V_{1}$.

Proof: This is a result from Lemma 3.2 and 3.3.

We use an example to illustrate our idea (Fig. 7). In Fig. 7(a), the distribution of the sensors are the same as in Fig. 5(a). We draw the boundaries of the unsafe regions for the 5 pairs of neighboring sensors (indicated by dotted circles) as in Lemma 3.3, and check if they intersect with $\overline{P Q}^{3}$. As can be seen, $\overline{P Q}$ intersects with the unsafe regions of $S_{2} S_{3}, S_{3} S_{4}$ and $S_{5} S_{1}$, and hence it is not full-view covered. Fig. 7(b) shows the case when there are four other sensors $S_{6}, S_{7}, S_{8}$ and $S_{9}$ covering $\overline{P Q}$. In this case, $\overline{P Q}$ can be fullview covered as no unsafe region intersects with it.

However, the ordered list $C L_{V}$ may not be the same for every point $V \in \overline{P Q}$. For example in Fig. $8, S_{1}$ is prior to $S_{2}$ in $V$ 's list, but $S_{2}$ is prior to $S_{1}$ in $U$ 's list. To resolve this issue, we partition $\overline{P Q}$ into sub-segments. For $1 \leq i \leq k-1$ and $i+1 \leq j \leq k$, if the line $S_{i} S_{j}$ intersects with $\overline{P Q}$, we mark the intersection point on $\overline{P Q}$. Then $\overline{P Q}$ is partitioned into sub-segments defined by every two adjacent marked points (including $P$ and $Q$ ). Since there are at most $k(k-1)$ intersection points, the total number of sub-segments is $O\left(k^{2}\right)$. Moreover, for a specific sub-segment $\overline{X Y}$, where $X$ and $Y$ are two adjacent marked points, all points on it have the same circular list of the sensors. Actually, if this is not true, there must be two points $U, V \in \overline{X Y}$, and two sensors $S_{1}, S_{2} \in S_{R}$, such that $S_{1}$ comes before $S_{2}$ in $V$ 's list but $S_{2}$ is before $S_{1}$ in $U$ 's list and there are no other sensors between them (Fig. 8). Then line $S_{1} S_{2}$ must have an intersection point with $\overline{P Q}$, between $X$ and $Y$, which is a contradiction to the fact that $X$ and $Y$ are adjacent intersection points.

Now we have a complete procedure for full-view coverage detection on a given segment of a sub-region's boundary. We can further apply this on all segments in $A$. For an estimation of the total running time, the whole region can be considered as a planar graph, where the vertices are the crossing points of sensing sectors and edges are the segments. As any two sensing sectors can have $O(1)$ crossing points on the perimeters, the number of vertices is $O\left(N^{2}\right)$, where $N$ is the total number of sensors. This further implies the total number of segments is $O\left(N^{4}\right)$. Our detection method requires $O\left(k^{2}\right)$ time on each segment, where $k(\leq N)$ is the number of sensors covering this segment. Therefore the total running time must be a polynomial function of $N$.

\footnotetext{
${ }^{3}$ In computation, this can be done by comparing the distance between the circle's center to $\overline{P Q}$ with the circle's radius.
}

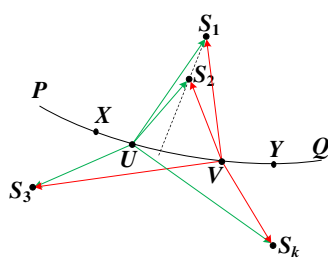

Fig. 8. The circular lists for $U, V$ are different: $C L_{V}=\left\{S_{1}, S_{2}, \ldots, S_{k}\right\}$ but $C L_{U}=\left\{S_{2}, S_{1}, \ldots, S_{k}\right\}$.

\section{Sensor Density Estimation for Full-View COVERAGE IN RANDOM DEPLOYMENT}

In this section, we derive an estimation on the lower bound of the probability that a region is full-view covered by a given number of randomly distributed sensors. With this result, we can estimate the sensor density needed to achieve full-view coverage with any given probability (e.g., 0.99).

\section{A. Technique Overview}

Consider a random uniform distribution of $N$ sensors in a square region $A$. Without loss of generality, we assume $A$ 's area is unit. Given $r, \varphi$ and $\theta$, we calculate the probability that $A$ is full-view covered. Generally, if sensors are deployed in a bounded region, the area very close to the boundary is likely to have fewer sensors than the interior area, and hence less likely to be covered as required. A common method to avoid this boundary effect is to deploy the sensors in a slightly larger region $A^{\prime}$, e.g., enlarging the side length of $A$ from $d$ to $d+r$ [5]. The difference is negligible if $A$ is sufficiently large. We can also make the analysis clean by assuming the sensor's coverage reflects at the boundary; i.e., for each sensor $S$ with distance less than $r$ to a boundary, we assume there is another sensor outside the boundary at the position symmetrical to $S$ with respect to the boundary. In the following analysis, we assume the boundary effect is negligible.

First we approximate the continuous region by discrete grid points. This is a common way to estimate the probability of area coverage [15]. We show that if the grids are sufficiently dense and are all full-view covered by a set of sensors with $\left(r^{\prime}, \varphi^{\prime}, \theta^{\prime}\right)$, where $r^{\prime}=r-\Delta r, \varphi^{\prime}=\varphi-\Delta \varphi$ and $\theta^{\prime}=\theta-\Delta \theta$ for any given $(\Delta r, \Delta \varphi, \Delta \theta)$, then the whole region is full-view covered by the same set of sensors with $(r, \varphi, \theta)$. Then we estimate a lower bound of the probability that all grid points are full-view covered. Based on this, we obtain a lower bound of the probability that $A$ is full-view covered.

In the following analysis, we first assume $\varphi=2 \pi$. This will give the essence of our method. Note that the major challenge of full-view coverage is due to the introduction of $\theta$, not $\varphi$. Then we extend the analysis for any $0<\varphi<2 \pi$. Note that in practice $\varphi=2 \pi$ can be considered as the case that each node is bundled with multiple camera sensors, facing to different directions to form a panoramic view. A camera that rotates around with negligible rotation time can also be considered as in this case.

\section{B. Probability Estimation for $\varphi=2 \pi$}

We use triangle lattices as the grids, although any other grid patterns may also suffice. Grid points are the vertices 


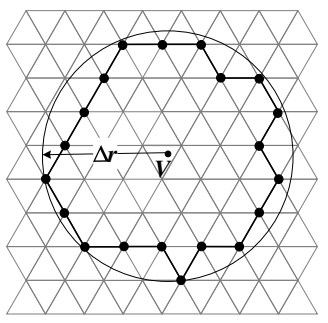

(a)

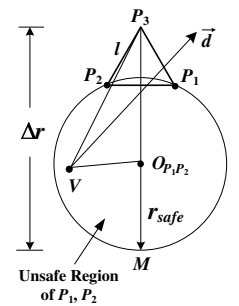

(b)
Fig. 9. (a) The black dots are edge points of $V$; (b) If $V$ is in the unsafe region of $P_{1}, P_{2}$, then $P_{3}$ is an edge point.

of equilateral triangles with side length $l$. Each grid point $P$ has six neighbors with distance $l$ from it (Fig. 9). They are called $P$ 's 1-hop neighbors. Given $A$ 's area fixed to be unit, the choice of $l$ depends on $(\Delta r, \Delta \theta)$.

Lemma 4.1 Given $(\Delta r, \Delta \theta)$, if $l \leq l_{0}(\Delta r, \Delta \theta)$, for any point $V \in A$ and any vector $\vec{d}$ from $V$, there is a grid point $P$ such that $\|V P\| \leq \Delta r$ and $\alpha(\vec{d}, \overrightarrow{V P}) \leq \Delta \theta$. Here $l_{0}(\Delta r, \Delta \theta)=\frac{2 \Delta r}{\sqrt{3}+\cot \Delta \theta}$.

Proof: Consider the set of all the grid points $P$ with $\|V P\| \leq \Delta r$, which is denoted by $G P_{V}(\Delta r)$. Define an edge point to be a grid point $P \in G P_{V}(\Delta r)$ such that $P$ has an 1-hop neighbor not in $G P_{V}(\Delta r)$ and an 1-hop neighbor in $G P_{V}(\Delta r)$.

All the edge points and the line segments connecting them form a polygon just inside the circle centered at $V$ with radius $\Delta r$ (Fig. 9(a)). Suppose the intersection point of vector $\vec{d}$ and the above polygon's boundary is between two neighboring edge points $P_{1}, P_{2}$. We claim $\alpha\left(d, V P_{1}\right)+\alpha\left(d, V P_{2}\right) \leq 2 \theta$, which will prove the lemma.

Suppose the claim is incorrect. Then from Lemma 3.3, $V$ is in the unsafe region of $P_{1}, P_{2}$, which means $\left\|V O_{P_{1} P_{2}}\right\|<$ $r_{\text {safe }}$, where $O_{P_{1} P_{2}}$ is the center of the circle defining the unsafe region (Fig. 9(b)). From trigonometry knowledge, we get $r_{\text {safe }}=\frac{l}{2 \sin (2 \Delta \theta)}$. So

$$
\left\|V O_{P_{1} P_{2}}\right\|<\frac{l}{2 \sin (2 \Delta \theta)}
$$

Consider the triangles with $P_{1} P_{2}$ as one side and a third vertex $P_{3} . P_{3}$ is either on the near side of $P_{1} P_{2}$ and closer from $V$ or on the far side of $P_{1} P_{2}$ and further from $V$. Consider the case when $P_{1}$ is on the far side. Then $\left\|V P_{3}\right\|>\Delta r$ (since if else, either $P_{1}$ or $P_{2}$ is not edge point).

On the other hand,

$$
\left\|P_{3} O_{P_{1} P_{2}}\right\|=r_{\text {safe }} \cdot \cos 2 \theta+\frac{\sqrt{3}}{2} l=\frac{l}{2}(\cot 2 \Delta \theta+\sqrt{3}) \text {. }
$$

If $l$ is as in the lemma, from triangle inequality

$$
\left\|V P_{3}\right\| \leq\left\|V O_{P_{1} P_{2}}\right\|+\left\|P_{3} O_{P_{1} P_{2}}\right\|<\Delta r .
$$

This is a contradiction. Thus the claim is proved.

Based on this result, we have the following condition regarding the whole region's coverage.

Lemma 4.2 Suppose $\varphi=2 \pi$ and all grid points are fullview covered by a set of sensors with $r^{\prime}=r-\Delta r$ and $\theta^{\prime}=\theta-$ $\Delta \theta$ for some given $(\Delta r, \Delta \theta)$. If $l \leq l_{0}(\Delta r, \Delta \theta)$ as indicated

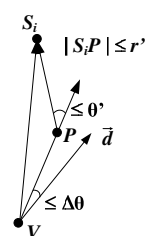

(a)

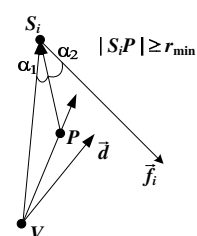

(b)
Fig. 10. Grid point property: (a) there is no constraint on the distance between $P$ and $S_{i}$ (Lemma 4.2); (b) there is a lower bound $r_{\min }$ on the distance between $P$ and $S_{i}$ (Lemma 4.5).

in Lemma 4.1, then any point $V \in A$ is full-view covered by the same set of sensors with $(r, \theta)$.

Proof: We need to prove that for any point $V \in A$ and any vector $\vec{d}$, there is a sensor $S_{i}$ such that $\left\|S_{i} V\right\| \leq r$ and $\alpha(\overrightarrow{V S}, \vec{d}) \leq \theta$.

Suppose $P$ is the grid point found in Lemma 4.1. Since $P$ is full-view covered, there is a sensor $S_{j}$ such that $\left\|P S_{j}\right\| \leq r^{\prime}$ and $\alpha\left(\overrightarrow{V P}, \overrightarrow{P S_{j}}\right) \leq \theta^{\prime}$ (Fig. 10(a)). From triangle inequality,

$$
\left\|V S_{j}\right\| \leq\|V P\|+\left\|P S_{j}\right\| \leq \Delta r+r^{\prime}=r .
$$

Thus $V$ is covered by $S_{j}$, and furthermore,

$$
\alpha\left(\vec{d}, \overrightarrow{V S_{j}}\right) \leq \alpha(\vec{d}, \overrightarrow{V P})+\alpha\left(\overrightarrow{V P}, \overrightarrow{V S_{j}}\right) \leq \Delta \theta+\theta^{\prime}=\theta .
$$

Thus $V$ is full-view covered by the sensors with $(r, \theta)$.

For any point $V \in A$, let $C_{V}$ denotes the event that $V$ is full-view covered.

Lemma 4.3 Suppose $\varphi=2 \pi$. Given $N$ sensors with $\left(r^{\prime}, \theta^{\prime}\right)$ uniformly distributed in $A$, the probability that a given point $V$ is full-view covered is

$\operatorname{Pr}\left(N, r^{\prime}, \theta^{\prime}\right) \triangleq \operatorname{Pr}\left[C_{V}\right]=\sum_{k=\frac{\pi}{\theta^{\prime}}}^{N}\left(\begin{array}{l}N \\ k\end{array}\right) p^{k}(1-p)^{N-k} f\left(k, \theta^{\prime}\right)$,

where $\pi / \theta^{\prime}$ is the abbreviation for $\left\lfloor\pi / \theta^{\prime}\right\rfloor, p=\pi r^{\prime 2}$ and

$$
f\left(k, \theta^{\prime}\right)=\sum_{j=0}^{\frac{\pi}{\theta^{\prime}}}\left(\begin{array}{l}
k \\
j
\end{array}\right)(-1)^{j}\left(1-j \frac{\theta^{\prime}}{\pi}\right)^{k-1} .
$$

Proof: For a uniformly distributed sensor $S_{i}$, the probability that it is within distance $r^{\prime}$ from $V$ is $p=\pi r^{\prime 2}$ and the probability that exactly $k$ sensors are within $r^{\prime}$ to $V$ is $\sum_{k=\frac{\pi}{\theta^{\prime}}}^{N}\left(\begin{array}{c}N \\ k\end{array}\right) p^{k}(1-p)^{N-k}$.

Consider the distribution of the sensor within the disk, since the sensor is uniformly distributed in $A$, its distribution is also uniform if conditioned on the disk area within distance $r^{\prime}$ to $V$. Furthermore, for each sensor $S_{i}$ within the disk, consider its projection $P_{i}$ on the perimeter of the circle centered at $V$ with radius $r^{\prime}$. It is the intersection point of vector $\overrightarrow{V S_{i}}$ and the circle. If we consider $P_{i}$ 's position on the circle, it is also uniformly distributed. From Lemma 3.2, given $k$ sensors within distance $r^{\prime}$ from $V$ (and hence able to cover $V$ ), $V$ is full-view covered if and only if the angle between any two adjacent vectors is no greater than $2 \theta$. This is equivalent to the event that the perimeter of a circle with unit length is covered by $k$ uniformly distributed arc segments with length $\theta^{\prime} / \pi$ (Fig. 11). The latter probability is given by $f\left(k, \theta^{\prime}\right)$, 


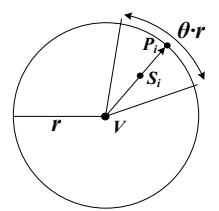

Fig. 11. $S_{i}$ 's coverage range is projected as an arc on the circle.

which is shown in [20]. Therefore we have the probability shown in the lemma.

From Lemma 4.2 and 4.3, we obtain a lower bound on the probability for region $A$ to be full-view covered.

Theorem 4.4 Given $r, \theta$ and $\varphi=2 \pi$, the probability that region $A$ is full-view covered by $N$ uniformly distributed sensors is lower bounded by $\operatorname{Pr}\left(N, \frac{\sqrt{N}-1}{\sqrt{N}} r, \frac{\sqrt{N}-1}{\sqrt{N}} \theta\right)^{M}$, where $\operatorname{Pr}(N, x, y)$ is given by Lemma 4.3, $M=\left\lceil\frac{8}{\sqrt{3}} l_{0}^{-2}\right\rceil$ and $l_{0}=l_{0}\left(\frac{r}{\sqrt{N}}, \frac{\theta}{\sqrt{N}}\right)$ is given by Lemma 4.1.

Proof: From Janson's Inequality [15] and Lemma 4.3, the probability that all grid points are full-view covered by $N$ sensors with $r^{\prime}=\frac{\sqrt{N}-1}{\sqrt{N}} r$ and $\theta^{\prime}=\frac{\sqrt{N}-1}{\sqrt{N}} \theta$ is no less than $\operatorname{Pr}\left(N, r^{\prime}, \theta^{\prime}\right)^{M}$, where $M$ is the number of grid points in a unit area. Then from Lemma 4.2, the whole area is full-view covered by sensors with $r=r^{\prime}+\Delta r$ and $\theta=\theta^{\prime}+\Delta \theta$, where $\Delta r=\frac{1}{\sqrt{N}} r$ and $\Delta \theta=\frac{1}{\sqrt{N}} \theta$, if the grid points are full-view covered by the same set of sensors with $\left(r^{\prime}, \theta^{\prime}\right)$. Therefore we have the lower bound shown as above.

\section{Probability Estimation for $\varphi<2 \pi$}

We use similar technique as above. Note that Lemma 4.1 and 4.2 are the keys to the establishment of the above result. The rationale behind it is that if the grid points are sufficiently dense and all full-view covered, then the whole region can be full-view covered if we slightly enlarge the sensor's radius and the effective angle. However, we assumed $\varphi=2 \pi$ there, which means any point $V$ within $\Delta r$ to a grid point $P$ can also been covered by the sensors that cover $P$, and hence makes the analysis clean. If $\varphi<2 \pi$, the sensor covering $P$ may not cover $V$ due to the sensor's orientation, no matter how close they might be to each other. A natural solution is to expand $\varphi^{\prime}$ to $\varphi$. However, it is difficult to guarantee a small bound on the increasing step $\left(\alpha_{1}\right.$ in Fig. 10(b)), and if we can not reasonably bound this value, the error of the estimation would be large. To overcome this difficulty, we require the grid points to be full-view covered by sensors which are at least certain distance (a tiny lower bound) away from it. Then we can establish similar results as in Lemma 4.1 and 4.2.

Lemma 4.5 Suppose each grid point can be full-view covered by sensors that are at least $r_{\min }$ distance away and with parameters $r^{\prime}=r-\Delta r, \theta^{\prime}=\theta-\Delta \theta$, and FoV angle $\varphi^{\prime}=\varphi-\Delta \varphi$, for some predefined $(\Delta r, \Delta \theta, \Delta \varphi)$. If $l \leq l(\Delta r, \Delta \theta, \Delta \varphi)$, then any point $A$ is full-view covered by the same set of sensors with $(r, \theta, \varphi)$. Here $l(\Delta r, \Delta \theta, \Delta \varphi)=$ $\frac{\min \left\{2 \Delta r, \Delta \varphi \cdot r_{\min }\right\}}{\sqrt{3}+\cot \Delta \theta}$.

Proof: We need to show that for any $V \in A$ and any facing direction (vector $\vec{d}$ ), there is a sensor $S_{i}$ such that $\left\|V S_{i}\right\| \leq r, \alpha\left(\overrightarrow{S_{i} V}, \overrightarrow{f_{i}}\right) \leq \varphi / 2$ and $\alpha\left(\overrightarrow{V S_{i}}, \vec{d}\right) \leq \theta$, where $\overrightarrow{f_{i}}$ is the orientation vector of $S_{i}$. We first observe that if $l$ is as indicated as above, it also satisfies the condition in Lemma 4.1. So there must be a grid point $P$ such that $\|V P\| \leq \min \left\{\Delta r, \Delta \varphi \cdot r_{\min } / 2\right\}$ and $\alpha(\vec{d}, \overrightarrow{V P}) \leq \Delta \theta$. Moreover, among the sensors that cover $P$, there must be a sensor $S_{i}$ such that $\left\|V S_{i}\right\| \leq r$ and $\alpha\left(\overrightarrow{V S_{i}}, \vec{d}\right) \leq \theta$. We only need to show that $\alpha\left(\overrightarrow{S_{i} V}, \overrightarrow{f_{i}}\right) \leq \varphi / 2$.

Note that $\alpha\left(\overrightarrow{S_{i} V}, \overrightarrow{f_{i}}\right) \leq \alpha_{1}+\alpha_{2}$, where $\alpha_{1}=\alpha\left(\overrightarrow{S_{i} V}, \overrightarrow{S_{i} P}\right)$ and $\alpha_{2}=\alpha\left(\overrightarrow{S_{i} P}, \overrightarrow{f_{i}}\right)$ (Fig. 10(b)). As $P$ is covered by $S_{i}, \alpha_{2} \leq \varphi^{\prime} / 2$. From trigonometry knowledge, we know $\alpha_{1} \leq \tan \alpha_{1}=\frac{\|V P\| \sin \beta}{\|V P\| \cos \beta+\left\|P S_{i}\right\|}$, where $\beta=\alpha\left(\overrightarrow{P S_{i}}, \overrightarrow{V P}\right)$. Notice that $\|V P\| \leq \Delta \varphi \cdot r_{\min } / 2,\left\|P S_{i}\right\| \geq r_{\min }$ and $\sin \beta \leq 1$. Therefore $\alpha_{1} \leq \Delta \varphi / 2$, and hence $\alpha\left(\overrightarrow{S_{i} V}, \overrightarrow{f_{i}}\right) \leq$ $\varphi^{\prime} / 2+\Delta \varphi / 2 \leq \varphi / 2$.

For any point $V \in A$, let $C_{V}^{r_{\min }}$ denote the event that $V$ is full-view covered by sensors which are at least $r_{\min }(<r)$ distance away.

Lemma 4.6 Given $N$ sensors with $\left(r^{\prime}, \theta^{\prime}, \varphi^{\prime}\right)$ uniformly distributed in region $A$, the probability for a given point $V$ to be full-view covered by sensors at least $r_{\min }\left(<r^{\prime}\right)$ away is

$$
\begin{aligned}
& \operatorname{Pr}\left(N, r_{\text {min }}, r^{\prime}, \theta^{\prime}, \varphi^{\prime}\right) \triangleq \operatorname{Pr}\left[C_{V}^{r_{\text {min }}}\right] \\
= & \sum_{s=\frac{\pi}{\theta^{\prime}}}^{N}\left(\begin{array}{c}
N \\
s
\end{array}\right) p^{s}(1-p)^{N-s} \sum_{k=\frac{\pi}{\theta^{\prime}}}^{s}\left(\begin{array}{l}
s \\
k
\end{array}\right) q^{k}(1-q)^{s-k} f\left(k, \theta^{\prime}\right),
\end{aligned}
$$

where $\pi / \theta^{\prime}$ is the abbreviation for $\left\lfloor\pi / \theta^{\prime}\right\rfloor, p=\pi\left(r^{\prime 2}-r_{\text {min }}^{2}\right)$, $q=\varphi^{\prime} / 2 \pi$, and $f\left(k, \theta^{\prime}\right)$ is as in Lemma 4.3.

Proof: First note that given a sensor $S_{i}$ with $r_{\min } \leq$ $\left\|V S_{i}\right\| \leq r^{\prime}$, since its orientation vector is uniformly distributed in $[0,2 \pi)$, the probability that $V$ is covered by $S_{i}$ is $q$. Also note that the probability that a sensor falls into the closed strip, with $r^{\prime}$ as outer radius and $r_{\min }$ as inner radius, is $p$. The meaning of $f\left(k, \theta^{\prime}\right)$ is the same as in Lemma 4.3. By combining these together, we have $\operatorname{Pr}\left[C_{V}^{r_{\text {min }}}\right]$ as shown above.

Now we can give a lower bound of the probability that $A$ is full-view covered.

Theorem 4.7 Given $(r, \theta, \varphi)$, the probability that region $A$ is full-view covered by $N$ uniformly distributed sensors is lower bounded by $\operatorname{Pr}\left(N, \frac{r}{\sqrt{N}}, \frac{\sqrt{N}-1}{\sqrt{N}} r, \frac{\sqrt{N}-1}{\sqrt{N}} \theta, \frac{\sqrt{N}-1}{\sqrt{N}} \varphi\right)^{M}$, where $\operatorname{Pr}(N, w, x, y, z)$ is given by Lemma 4.6, $M=\left\lceil\frac{8}{\sqrt{3}} l^{-2}\right\rceil$ and $l=l\left(\frac{r}{\sqrt{N}}, \frac{\theta}{\sqrt{N}}, \frac{\varphi}{\sqrt{N}}\right)$ is given by Lemma 4.5.

Proof: From Lemma 4.5 and 4.6, this can be proved by following the same argument as in Theorem 4.4.

\section{Sensor Density Estimation for Full-View Coverage in Deterministic Deployment}

Deterministic deployment is the best way to achieve fullview coverage in a controlled environment, e.g., in indoor surveillance where camera sensors can be placed at any place as required. In traditional disk model, triangle lattice based deployment is proved to be optimal in terms of sensor density [13]. In this section, we construct a deployment pattern for full-view coverage based on triangle lattice. We show a necessary and sufficient condition on the grid length such that the whole area can be full-view covered. Based on that, we 


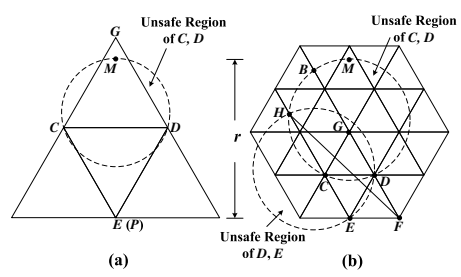

Fig. 12. Necessary condition for triangle lattice: (a) $\theta \geq \pi / 6$; (b) $\theta<\pi / 6$.

derive an estimation on the sensor density needed for fullview coverage in the triangle lattice based deployment and show that it is at most a factor from the optimal deployment pattern.

\section{A. Triangle Lattice}

The triangle lattice is constructed as follows. First we place $\lceil 2 \pi / \varphi\rceil$ sensors together on a single point and let them face different directions to form a single node with $\varphi=2 \pi$. Then we place the sensor nodes on the vertices of the equilateral triangles with grid length $l$. Region $A$ has unit area and it is assumed to be sufficiently large compared with $r$ and hence we ignore the boundary effect in deployment.

\section{B. Density Estimation for Triangle Lattice Based Deployment}

The grid length $l$ of the triangle is critical. If it is too large, there will be points not full-view covered. If it is too small, the deployment density and hence the cost may be too high. Given the sensor radius $r$ and the effective angle $\theta$, we want to calculate the best $l$ such that every point in $A$ is full-view covered.

Actually if we replace $(\Delta r, \Delta \theta)$ by $(r, \theta)$ in Lemma 4.1, we immediately have a sufficient condition on $l$.

Lemma 5.1 Suppose sensors are deployed on the vertices of the triangle lattices with grid length $l$. Given $(r, \theta)$, if $l=$ $l(r, \theta)=\frac{2 r}{\sqrt{3}+\cot \theta}$, then every point in $A$ is full-view covered.

Proof: This is a direct result from Lemma 4.1.

In fact, this is also a necessary condition for full-view coverage in the triangle lattice based deployment.

Lemma 5.2 If region $A$ is full-view covered, the grid length should be no smaller than $l=l(r, \theta)=\frac{2 r}{\sqrt{3}+\cot \theta}$.

Proof: There are two cases: $\theta \geq \pi / 6$ and $\theta<\pi / 6$. If $\theta \geq$ $\pi / 6$, consider the situation in Fig.12(a). $M$ is the intersection point of $E G$ and the boundary of the unsafe region of $C, D$, which is a portion of the circle centered at $O_{C, D}$. Let $V$ be a point on the segment $E M$ and with distance $\epsilon(>0)$ to $M$. Let $r^{\prime}=\|E V\|$. Since $V$ is in the unsafe region of $C, D$, which means $\angle C V D>2 \theta$, there must be a grid point $P$ such that either $\angle C V P<2 \theta$ or $\angle D V P<2 \theta$ and $P$ can cover $V$. This can only happen if $r \geq r^{\prime}$ (and hence $P$ is $E$ ) because if not, there would be no grid point between line $V C$ and $V D$ which can cover $V$. Let $\epsilon \rightarrow 0$ and hence $r \rightarrow r^{\prime}=\|E M\|$, which implies the critical value of $l$.

If $\theta<\pi / 6$, consider the situation in Fig.12(b). In this case, the boundary of the unsafe region of $C, D$ intersects with line $E C$ on $H$ and intersects with line $D G$ on $B$. First we notice that $H$ is also the intersection point of the boundary of the unsafe region of $E, D$ and line $E C$. In fact, if we denote

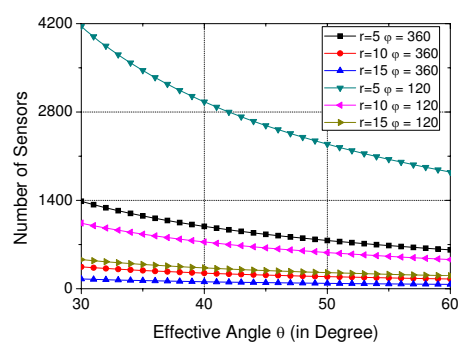

Fig. 13. Number of sensors in triangular lattice based deployment.

this intersection point by $H^{\prime}$, then $\angle E H^{\prime} D=2 \theta$ according to Lemma 3.3. Similarly, $\angle E H D$ also equals to $2 \theta$. Since $H$ and $H^{\prime}$ are on the same line, they are the same point. Then since $H E$ is parallel to $B D,\|H B\|=\|D E\|$, which further equals to $\|C D\|$ and $\|C G\|$. Thus polygon $H C G B$ is a parallelogram, which means $\|H C\|=\|B G\|$. From this, we know $\triangle H C F$ and $\triangle B G E$ are congruent triangles, which means $\|H F\|=\|B E\|$. By a similar argument as in the above case, we know that if $r$ is smaller than $\|H F\|$, there is a point sufficiently close to $H$ such that it is not full-view covered. Now consider the case when $\theta \rightarrow 0$. This implies $\|H F\|=$ $\|B E\| \rightarrow\|B G\|$ and $\|B G\| \rightarrow\|E M\|(\rightarrow \infty)$, which further implies $\|H F\|=\|E M\|$ and hence we have the critical value of $l$.

From the critical value of $l$ obtained above, we calculate the required sensor density for the triangle lattice based deployment. We compare it with other possible deployment patterns.

Theorem 5.3 Given $(r, \theta, \varphi)$, the sensor density for the triangle lattice based deployment is $\frac{\pi}{\varphi\left|A_{l}\right|}$ which is no more than $\frac{\theta r^{2}}{2\left|A_{l}\right|}$ of the density of any other deployment patterns. Here $\left|A_{l}\right|=\frac{\sqrt{3} r^{2}}{3+2 \sqrt{3} \cot \theta+(\cot \theta)^{2}}$, which is the area of a equilateral triangle with side length $l$.

Proof: First, from Lemma 5.1 we know $l$ and hence the area of each triangle with side length $l$, which is exactly $\left|A_{l}\right|$ shown as above. Then note that each triangle has 3 vertices, and each vertex is the intersection point of 6 triangles. Thus the total number of grid points in a unit area region is $\frac{|A|}{\left|A_{l}\right|} \cdot \frac{3}{6}=$ $\frac{1}{2\left|A_{l}\right|}$. Thus the total number of sensors needed is $\frac{2 \pi}{\varphi} \cdot \frac{1}{2\left|A_{l}\right|}=$ $\frac{\pi}{\varphi\left|A_{l}\right|}$.

On the other hand, for any deployment patterns, each point in $A$ should be covered by at least $\pi / \theta$ sensors. Note that each sensor can only cover $\varphi r^{2} / 2$ area of $A$, which is the area of the sensing sector. Thus the total number of sensor needed is at least $\frac{\pi / \theta}{\varphi r^{2} / 2}=\frac{2 \pi}{\theta \varphi r^{2}}$.

Finally, the ratio of the above two values yields the bound on the scaling factor in the theorem.

Figure 13 is an illustration on the number of sensors needed for full-view coverage in an $100 \mathrm{~m} \times 100 \mathrm{~m}$ field when triangular lattice based deployment is used $\left(\theta\right.$ is from $\frac{\pi}{6}$ to $\frac{\pi}{3}$, for $r=5,10,15$ and $\varphi=\frac{2}{3} \pi, 2 \pi$ respectively).

\section{Discussion}

Finally, we make some remarks on the orientation of camera sensors. In practice, some high power cameras may periodically rotate around, and hence cover more area than 

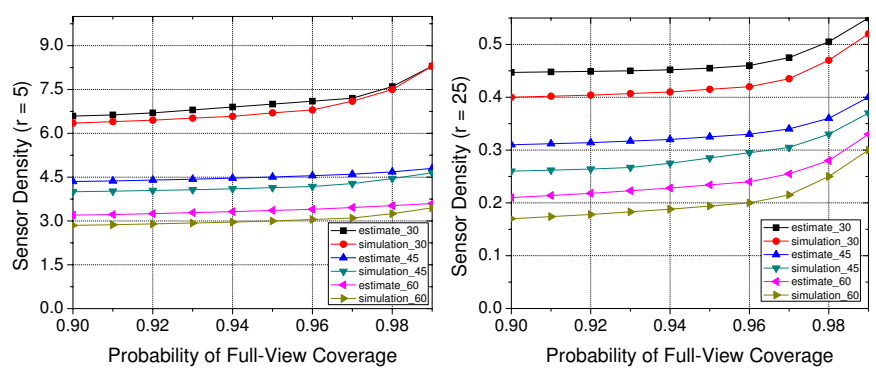

Fig. 14. Density vs. Probability: $r=5$ (left) and $r=25$ (right).

static cameras. If the rotation time is negligible compared with the object's moving speed, the camera can be considered as having $2 \pi$ FoV angle, and hence our results in this section and Section IV-B can be applied directly. If the rotation time is non-negligible, coverage delay may become another factor that have impact on the quality of coverage [24]. Further study is needed to understand the performance regarding the effects of both viewing direction and coverage delay.

\section{Simulation AND Numerical Results}

In this section, we show some simulation results on fullview coverage. The purpose of the simulation is two-fold. First we want to validate the theoretical results on sensor density estimation for full-view coverage. Second we want to have a pictorial view of the relationship between sensor density and the percentage of full-view coverage.

\section{A. Simulation Setup}

The target field $A$ is a $100 m \times 100 m$ square region. We use two settings for sensing radius: $r=5 \mathrm{~m}$ and $r=25 \mathrm{~m}$. In both cases, we deploy the sensors in the field with area of $(100+$ $2 r) m \times(100+2 r) m$ to circumvent the boundary effect. The difference is that when $r$ is $5 \mathrm{~m}$, it is much smaller compared with the side length and hence the deployment field is almost the same as $A$. But if $r=25 m$, it is comparable to the side length and hence the density results (both in the simulation and theoretical estimation) are for the enlarged deployment field. The FoV angle is fixed to be $\varphi=\pi / 3$, and we use three values for the effective angle, i.e., $\theta=\pi / 6, \pi / 4, \pi / 3$ (or $30,45,60$ in degree) respectively.

In the first part of the simulation, we vary the number of sensors from 10000 to 90000 for $r=5 m$, and from 1000 to 6000 for $r=25 \mathrm{~m}$, to observe the full-view coverage probability. We adopt the methodology as in [15] to calculate the probability in simulation. Each experiment is run 100 times, and the results are averaged. As comparisons, we also give the theoretical estimation for each configuration. Note $r$ is normalized to 0.05 and 0.25 respectively.

In the second part of the simulation, we vary the number of sensors from 4000 to 40000 for $r=5 \mathrm{~m}$, and from 200 to 2000 for $r=25 \mathrm{~m}$, to observe the percentage of full-view coverage. The percentage of full-view coverage is defined to be the percentage of points that are full-view covered. Each result shown here is the statistical average of 100 experiments.
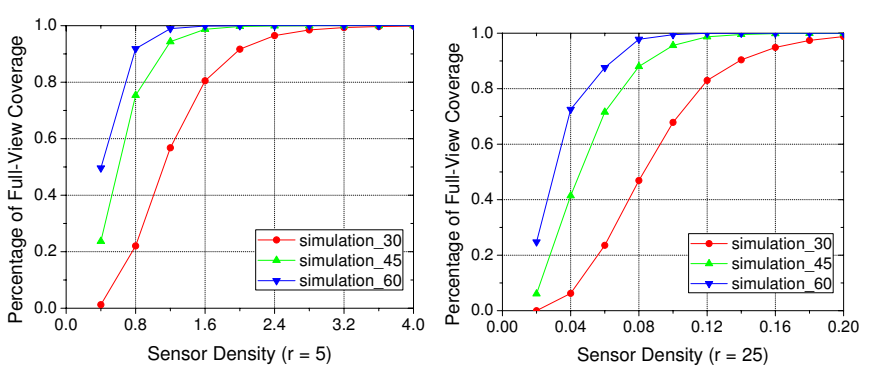

Fig. 15. Percentage vs. Density: $r=5$ (left) and $r=25$ (right).

\section{B. Simulation Result Analysis}

Figure 14 shows the results of the sensor density under different probability requirement for full-view coverage. We use $x$-axle to denote the probability and $y$-axle to denote the sensor density. The results shown here are for probability requirement above 0.9 , which would be of more interest in practice. The sensor density is normalized by dividing the total number of sensors by the target field' area. The results shown here are for $r=5$ and $r=25$. In both cases, the sensor density needed for full-view coverage increases as the required probability increases, although the density for $r=25$ is much lower than the density for $r=5$ (reflected by the range on $y$-axle). The theoretical results (indicated by 'estimate' in the figures) serve as upper bounds for the real densities (indicated by 'simulation' in the figures) in all cases, which means as long as the sensor density reaches the theoretical bound, the coverage probability is guaranteed. Moreover, the theoretical bounds are very close to the real deployment density. The difference becomes even smaller as the required probability is higher. This further validates the theoretical estimation.

Figure 15 shows the results on the percentage of full-view coverage under different sensor densities. The percentage of full-view covered points increases very quickly as the sensor density increases. By comparing this figure and Figure 14, we can see that although the density needed to achieve fullview coverage for the whole target field may be high, the density needed for a high percentage (but not $100 \%$ ) of fullview coverage is much lower. For example, when $\theta=\pi / 4$ and $r=25,90 \%$ of the field is full-view covered when the density is around 0.1 (1000 sensors). But if we want to achieve full-view coverage for the whole area with probability 0.9 , the density should be above 0.25 ( 2500 sensors).

\section{RELATED WORK}

Coverage problem under disk sensing model has been studied extensively in the past few years. More comprehensive surveys on coverage detection (verification) methods can be found in [1], [9]. Here we only review the most relevant work. In [11], it is shown that an area is $k$-covered if and only if the perimeter of all sensor's sensing range (disk) is $k$-covered. A polynomial-time detection algorithm has been proposed based on this perimeter coverage property. In [6], the idea of perimeter coverage has been developed into a distributed protocol in which no location but only distance information is assumed to be known by the sensors. Based on the same assumption, Kasbekar et al. [12] show that the target field is 
$k$-covered if the intersection points of the perimeter of any two sensors' sensing disks are $k$-covered. They also present a distributed protocol which schedules the sensors to prolong the lifetime of the network with coverage guarantee. Another direction on coverage detection is to utilize the property of the Voronoi Diagram. Some interesting works are [10], [22], etc.

Sensor density estimation for $k$-coverage has been studied in [14], [25]. In [14], three kinds of deployments, square grid, uniform distribution and poisson distribution, are considered. Each sensor is assumed to work with probability $p$. Critical condition on the relationship among the number of sensors, sensing radius and the working probability $(p)$ for the target field to be almost surely $k$-covered has been established. As people realized that requiring the whole target field to be $k$ covered (full coverage) is not always necessary, various models on partial coverage are proposed. Barrier coverage [14] and trap coverage [5] are two such variants. In barrier coverage, the sensing disks of the active sensors form a strip zone which serves as a barrier, and any object crossing the target field is supposed to be detected by the sensors on the barrier. In trap coverage, coverage holes are allowed to exist as long as the diameters of the holes are bounded. Sensor density estimation for these coverage requirements are derived [14], [4], [16], [5]. The optimal deterministic deployment pattern for 1-coverage is based on triangle lattices, which has been proved in [13]. One of the latest results on achieving both coverage and connectivity in deterministic deployment under disk model can be found in [3].

The above studies under disk coverage model inspire our work in this paper. The major difference between theirs and ours is that full-view coverage requires consideration of three factors: the distance between the point and the sensor, the viewing direction of the sensor, and the orientation of the sensor, while in disk model, only the distance needs to be considered. All these issues make the full-view coverage problem much more complicated and challenging.

\section{CONCLUSION}

Camera sensor network has drawn much attention in the research community due to its huge potential in many applications. One fundamental research issue in camera sensor network is how to define coverage. Since traditional disk sensing model does not address the issue of viewing direction, which is intrinsic to camera sensors, we introduce a novel model called full-view coverage. A target field is said to be full-view covered if for any point $V$ and an arbitrary facing direction (i.e., a vector $\vec{d}$ ), there is always a sensor $S_{i}$ such that $V$ is in $S_{i}$ 's sensing range and the angle between $\vec{d}$ and the direction vector $\overrightarrow{V S}$ is smaller than a predefined value $\theta$. With this model, we propose an efficient method for full-view coverage detection in any given camera sensor network. We also derive a sufficient condition on the sensor density needed for full-view coverage in a random uniform deployment. Finally, we show a necessary and sufficient condition on the sensor density for full-view coverage in a triangular lattice based deployment.
The results in this paper can be used to evaluate the coverage of any deployed camera sensor networks where a deterministic guarantee on the detection result is desired, and the results also provide a guideline for real deployment of large scale camera sensor networks.

\section{REFERENCES}

[1] N. Ahmed, S. S. Kanhere, and S. Jha. The holes problem in wireless sensor networks: a survey. ACM SIGMOBILE Mob. Comput. Commun. Rev., 9(2):4-18, 2005.

[2] I. F. Akyildiz, T. Melodia, and K. R. Chowdhury. A survey on wireless multimedia sensor networks. Comput. Netw., 51(4):921-960, 2007.

[3] X. Bai, Z. Yun, D. Xuan, T.-H. Lai, and W. Jia. Optimal patterns for four-connectivity and full coverage in wireless sensor networks. IEEE Trans. Mob. Comput., 9(3):435-448, 2010.

[4] P. Balister, B. Bollobas, A. Sarkar, and S. Kumar. Reliable density estimates for coverage and connectivity in thin strips of finite length. In ACM MobiCom 2007.

[5] P. Balister, Z. Zheng, S. Kumar, and P. Sinha. Trap coverage: Allowing coverage holes of bounded diameter in wireless sensor networks. In IEEE INFOCOM 2009.

[6] Y. Bejerano. Simple and efficient k-coverage verification without location information. In IEEE INFOCOM 2008

[7] V. Blanz, P. Grother, P. J. Phillips, and T. Vetter. Face recognition based on frontal views generated from non-frontal images. In Proceedings of the 2005 IEEE Computer Society Conference on Computer Vision and Pattern Recognition (CVPR'05), 2005.

[8] Y. Cai, W. Lou, M. Li, and X.-Y. Li. Energy efficient target-oriented scheduling in directional sensor networks. IEEE Trans. Comput., 58(9):1259-1274, 2009.

[9] M. Cardei and J. Wu. Energy-efficient coverage problems in wireless ad-hoc sensor networks. Comput. Commun., 29(4):413-420, 2006.

[10] B. Cărbunar, A. Grama, J. Vitek, and O. Cărbunar. Redundancy and coverage detection in sensor networks. ACM Trans. Sen. Netw., 2(1):94$128,2006$.

[11] C.-F. Huang and Y.-C. Tseng. The coverage problem in a wireless sensor network. In ACM WSNA 2003.

[12] G. S. Kasbekar, Y. Bejerano, and S. Sarkar. Lifetime and coverage guarantees through distributed coordinate-free sensor activation. In $A C M$ MobiCom 2009.

[13] R. Kershner. The number of circles covering a set. American Journal of Mathematics, 61(3):665-671, 1939.

[14] S. Kumar, T. H. Lai, and A. Arora. Barrier coverage with wireless sensors. In ACM MobiCom 2005.

[15] S. Kumar, T. H. Lai, and J. Balogh. On k-coverage in a mostly sleeping sensor network. In ACM MobiCom 2004.

[16] B. Liu, O. Dousse, J. Wang, and A. Saipulla. Strong barrier coverage of wireless sensor networks. In ACM MobiHoc 2008.

[17] P. J. Phillips, W. T. Scruggs, A. J. O’Toole, P. J. Flynn, K. W. Bowyer, C. L. Schott, and M. Sharpe. FRVT 2006 and ICE 2006 large-scale results. National Institute of Standards and Technology, Tech. Rep. NISTIR 7408, 2007.

[18] B. Rinner and W. Wolf. A bright future for distributed smart cameras. Proceedings of the IEEE, 96(10):1562 - 1564, 2008.

[19] C. Sanderson, T. Shang, and B. C. Lovell. Towards pose-invariant 2d face classification for surveillance. In $A M F G$ '07: Proceedings of the $3 r d$ international conference on Analysis and modeling of faces and gestures, pages 276-289, 2007.

[20] H. Solomon. Geometric Probability. Society for Industrial and Applied Mathematics (SIAM), Philadelphia, PA, USA, 1978.

[21] P.-J. Wan and C.-W. Yi. Coverage by randomly deployed wireless sensor networks. IEEE/ACM Trans. Netw., 14(SI):2658-2669, 2006.

[22] G. Wang, G. Cao, and T. F. La Porta. Movement-assisted sensor deployment. IEEE Transactions on Mobile Computing, 5(6):640-652, 2006.

[23] P. Wang, R. Dai, and I. F. Akyildiz. Collaborative data compression using clustered source coding for wireless multimedia sensor networks. In IEEE INFOCOM 2010.

[24] Y. Wang and G. Cao. Minimizing service delay in directional sensor networks. In IEEE INFOCOM 2011.

[25] H. Zhang and J. Hou. On deriving the upper bound of $\alpha$-lifetime for large sensor networks. In ACM MobiHoc 2004. 